\title{
Successful management of a giant angiomyolipoma with partial nephrectomy
}

\author{
Lim EJ, Consigliere DT, Tiong HY * \\ Department of Urology, National University Health System, Singapore
}

Received: July 13, 2015

Accepted: September 1, $2015 \quad$ Online Published: September 6, 2015

DOI: $10.5430 /$ css.v2n1p19

URL: http://dx.doi.org/10.5430/css.v2n1p19

\begin{abstract}
Renal angiomyolipoma (AML) is largely defined as a benign tumor with 3 components: adipose tissue, abnormally thick-walled blood vessels and smooth muscle. Varying sizes of AML has been reported in the literature, with the general consensus that a size greater than $10 \mathrm{~cm}$ can be termed a giant AML and are associated with increased complications. This report discusses a case of giant AML with a diameter of up to $24 \mathrm{~cm}$ that underwent a successful partial nephrectomy without prior embolization.
\end{abstract}

Key Words: Giant angiomyolipoma, Partial nephrectomy, Renal angiomyolipoma

\section{INTRODUCTION}

Renal angiomyolipoma (AML) is largely defined as a benign tumor with 3 components: adipose tissue, abnormally thick-walled blood vessels and smooth muscle. Varying sizes of AML have been reported in the literature, with the general consensus that a size greater than $10 \mathrm{~cm}$ can be termed a giant AML. The associated increased complications of a giant AML include life threatening hemorrhage into the retroperitoneum or the renal collecting system, compression by virture of its mass and refractory pain. Previous literature has found a correlation of AML size to an increased presentation of the typical triad of symptoms (flank pain, palpable tender mass, hematuria), with a $52 \%-91 \%$ prevalence of hemorrhage or other symptoms with AML $>4 \mathrm{~cm} .{ }^{[1]}$ This report discusses a case of giant AML with a diameter of up to $24 \mathrm{~cm}$ that underwent a successful partial nephrectomy without prior embolization.

\section{Case presentation}

A 28-year-old woman presented with a 2 year history of right loin pain on a known background of AML. Her renal func- tion was unimpaired with a serum creatinine of $55 \mu \mathrm{mol} / \mathrm{L}$ and an eGFR of more than $60 \mathrm{ml} / \mathrm{min}$. Based on computed tomography (CT) scans performed in other institutions, the dimensions of her right giant AML were that of $24.9 \mathrm{~cm} \times$ $13.2 \mathrm{~cm} \times 13.5 \mathrm{~cm}$ (see Figure $1 \mathrm{a}, \mathrm{b}, \mathrm{c}$ ). There was significant vascularity arising from the upper pole of the kidney and the kidney was rotated medially by the enveloping AML (see Figure $1 \mathrm{~d}$ ). Following detailed consultation with the patient regarding the possible treatment options of angioembolization and open partial nephrectomy, the patient elected for the latter. With the patient in a full flank lateral posture, an extraperitoneal incision was made through the $11^{\text {th }}$ rib. Intraoperative findings were consistent with CT scan findings of a large mass arising from multiple foci in the upper pole. The mass enveloped the kidney and hilum, displacing the lower pole anteriorly. After the initial mobilization of the inferior pole of the kidney and the mass, the problem of accessing the hilum arose. Although an attempt was made to deliver the kidney and AML through the wound to access the hilum inferiorly, this was not possible due to large bulk. Instead, the tumour was mobilized and

\footnotetext{
* Correspondence: Tiong HY; Email: surthy@ nus.edu.sg; Address: Department of Urology, National University Health System, NUHS Tower Block, Level 8, 1E Kent Ridge Road, 119228, Singapore.
} 
de-bulked away from the renal parenchyma along avascular tissue planes, with residual main attachments to its origin in the upper pole. This provided access to the kidney hilum, which was then clamped en bloc with statinsky clamps after the administration of IV mannitol. The giant AML had 3 separate foci that was excised from the upper pole of the kidney along with the rest of the bulk with cold scapula in- cisions. The ureter was dealt with cautiously, and repair of the renal defect was performed with interrupted 2/0 vicryl interrupted sutures over Floseal Hemostatic Matrix (Baxter) and Surgicel ${ }^{\circledR}$ Fibrillar ${ }^{\circledR}$ Absorbable Hemostat (Ethicon) after figure of eight $4 / 0$ vicryl sutures to control medullary vasculature (see Figure 2). Total warm ischemic time was 18 minutes; total blood loss was $800 \mathrm{mls}$.
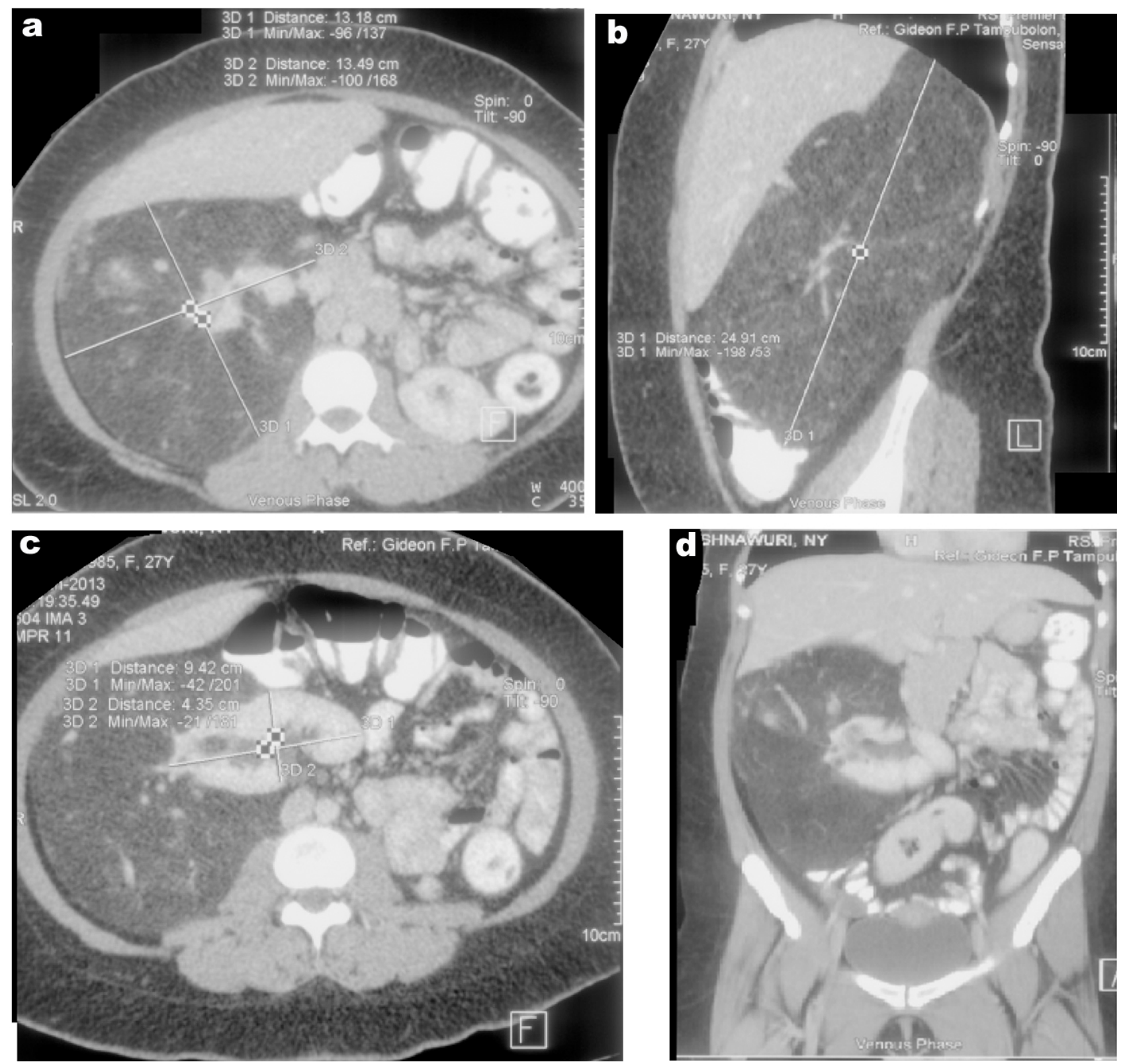

Figure 1. Enhanced Computed Tomography Urogram demonstrating dimensions of the AML a: Enhanced CTU of the AML, transverse view; $b$ : Enhanced CTU of the AML, sagittal view; $c$ : Enhanced CTU, transverse view, demonstrating displacement of kidney; $d$ : Enhanced CTU, coronal view

There were no major post operative complications, with a 1 unit transfusion of blood for a hemoglobin drop. Otherwise, her pain control was adequate with patient-controlled analgesia (PCA), with drain removal performed after four days. She was discharged six days post op with preserved renal function; creatinine of $60 \mu \mathrm{mol} / \mathrm{L}$ and an eGFR of more 
than $60 \mathrm{ml} / \mathrm{min}$. No residual nor recurrent disease was detected on a repeat CT urography done 5 months later (see Figure 3).

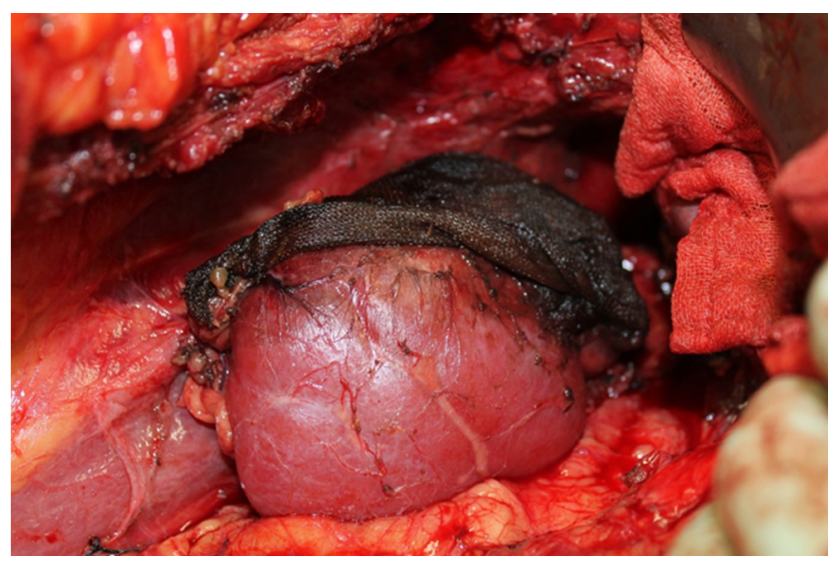

Figure 2. Intra-operative image of preserved kidney after resection of AML

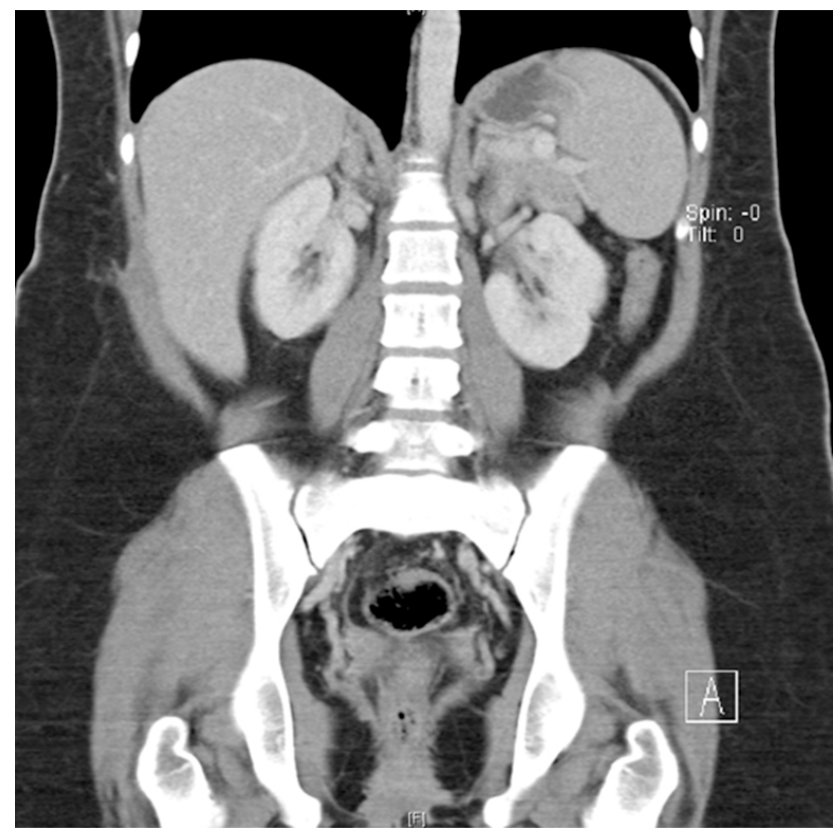

Figure 3. Enhanced CTU five months after partial nephrectomy

Histopathology showed fleshy yellow masses measuring $19.5 \mathrm{~cm} \times 11.5 \mathrm{~cm} \times 6.0 \mathrm{~cm}, 22.0 \mathrm{~cm} \times 9.0 \mathrm{~cm} \times 5.0 \mathrm{~cm}$ and $10.5 \mathrm{~cm} \times 13.0 \mathrm{~cm} \times 6.0 \mathrm{~cm}$ with the smaller fragment aggregating $15.0 \mathrm{~cm} \times 4.0 \mathrm{~cm} \times 5.0 \mathrm{~cm}$. Total weight was 1,700 g (see Figure 4). Microscopic sections showed cells with a predominant adipose tissue component and minor vascular and spindle cell components with no areas of sarcomatoid change or epithelioid pattern identified.

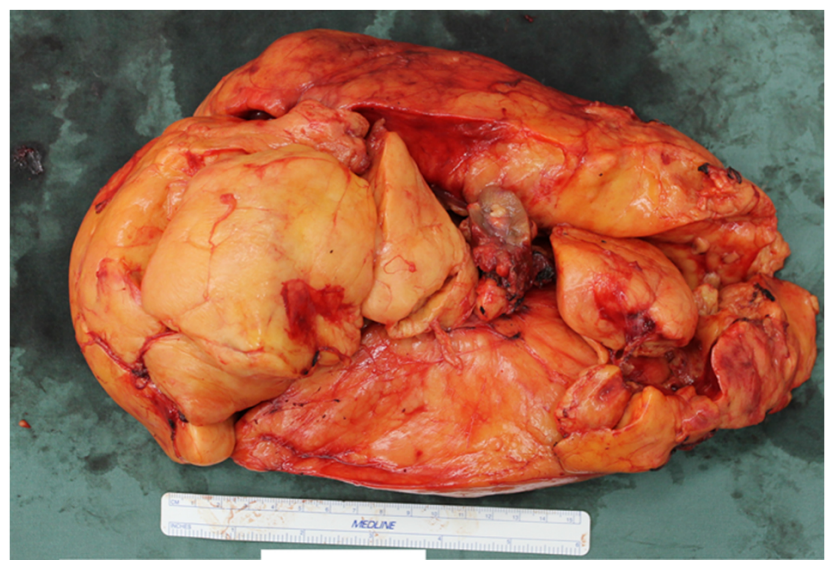

Figure 4. Macroscopic appearance of AML

\section{Discussion}

In considering the management of AMLs, the main aims are to prevent major hemorrhage while preserving renal function. For patients with asymptomatic small tumors that are less than $4 \mathrm{~cm}$, interval imaging as a conservative option is preferred. Conversely, intervention is considered mainly if the tumor is larger and symptomatic. This stems from the established relationship between size of the tumor, aneurysm formation and spontaneous rupture; tumors larger than $4 \mathrm{~cm}$ and aneurysms greater than or equal to $5 \mathrm{~mm}$ in relation to size had a higher probability of rupture. ${ }^{[2]}$ Thus, therapeutic treatment is recommended with larger tumors having a greater risk of spontaneous or traumatic rupture and hence subsequent hemorrhagic complications.

Treatment modalities can be mainly divided into transcatheter arterial embolization (TAE) or surgical intervention. Studies have shown that TAE has some long-term efficacy in the treatment of AML, reducing the need for surgical intervention and subsequent hemorrhagic complications, mainly via blocking the blood supply to the AML and thus reducing hemorrhagic risk. In reviewing the literature, there are few reports of giant AMLs managed with TAE; it must be noted that the size of the AMLs in these studies ranged from $10 \mathrm{~cm}-15 \mathrm{~cm}$. Most studies did not report a significant decrease in tumor size, with recurrence of the tumor and repeat embolization required. ${ }^{[3]}$ Preoperative TAE has been suggested to possibly reduce blood loss intraoperatively, but it must be weighed up with the possible complications following TAE. Reported decrease in size after angioembolization is relatively modest at thirty percent to fifty percent. ${ }^{[4]}$ In this young patient, TAE would have resulted in an inadequate reduction of size to its greater risk of subsequent hemorrhage complications. In addition, post embolization inflammatory reaction for such a large tumor was a consideration for us in opting for partial nephrectomy. Two previously reported gi- 
ant AML cases treated with TAE showed that although TAE was possible, post treatment both patients required multiple hospital admissions and transfusions, with one case eventually requiring a further partial nephrectomy. ${ }^{[5]}$ A systemic review of 524 cases showed that TAE of angiomyolipomas demonstrates low rates of mortality but with serious complications; with the most common complications being the need for revascularization in up to $30 \%$ of the cases, and recurring or refractory symptoms in $16.7 \%$. ${ }^{[6]}$ There have not been large studies published in regards to nephron sparing surgery in the approach to angiomyolipoma; however case series and case reports have reported low complication rates of up to $12 \%$ with low local recurrence rates. ${ }^{[7]}$ Specifically with regards to giant AML, there has been another case report which was also managed through a partial nephrectomy without prior embolization, and showed good recovery with a short hospital stay. ${ }^{[8]}$

In conclusion, the management of giant AMLs is ultimately patient-centric and tailored on a case-by-case basis, and even for large tumors, partial nephrectomy remains a viable option that should be considered. This case report has demonstrated that in such a case of giant AML with its localized foci in the upper pole of the kidney, partial nephrectomy is not just possible, but indeed could be the better treatment of choice.

\section{REFERENCES}

[1] Nelson CP, Sanda MG. Contemporary diagnosis and management of renal angiomyolipoma. J Urol. 2002; 168: 1315-1325. http: //dx.doi.org/10.1016/S0022-5347 (05) 64440-0

[2] Yamakado K, Tanaka N, Nakagawa T, et al. Renal angiomyolipoma: relationships between tumor size, aneurysm formation, and rupture. Radiology. 2002 Oct; 225(1): 78-82. PMid: 12354988. http: //dx.doi.org/10.1148/radiol.2251011477

[3] Schwartz MJ, Smith EB, Trost DW, et al. Renal artery embolization:clinical indications and experience from over 100 cases. BJU Int. 2007 Apr; 99(4): 881-6. PMid: 17166242. http://dx.doi.org/1 $0.1111 / \mathrm{j} .1464-410 \mathrm{X} .2006 .06653 . \mathrm{x}$

[4] Sooriakumaran P, Gibbs P, Coughlin G, et al. Angiomyolipomata: challenges, solutions, and future prospects based on over 100 cases treated. BJU Int. 2010 Jan; 105(1): 101-6. PMid: 19493268. http: //dx.doi.org/10.1111/j.1464-410X.2009.08649.x
[5] Cranston DW, Moslim M. CASE REPORTS: Unusual Giant Renal Angiomyolipoma (Renal Hamartoma): Clinical manifestation, Radiology, Pathology and Treatment. BJUI Int. 2012. Available from: http://shar.es/QX7UJ

[6] Murray TE, Doyle F, Lee M. Transarterial Embolization of Angiomyolipoma: A Systemic Review. J Urol. 2015 Sep; 194(3): 635-9. PMid: 25916674. http://dx.doi.org/10.1016/j . juro. 2015 .04 .081

[7] Boorjian SA, Frank I, Inman B, et al. The role of partial nephrectomy for the management of sporadic renal angiomyolipoma. Urology. 2007 Dec; 70(6): 1064-8. PMid: 18158015. http://dx.doi.org /10.1016/j.urology.2007.07.045

[8] Coskuner ER, Ozkan B, Yalcin V. The Role of Partial Nephrectomy without Arterial Embolization in Giant Renal Angiomyolipoma. Case Rep Med. 2012; 2012: 365762. http://dx. doi .org/10.1155/2 $012 / 365762$ 